\title{
The BioMAX beamline for macromolecular crystallography at MAX IV
}

\author{
Ana Gonzalez, Oskar Aurelius, Monika Bjelčić, Mikel Eguiraun, Ishkhan Gorgisyan, Elmir Jagudin, Sandesh \\ Kanchugal, Tobias Krojer, Mirko Milas, Jie Nan, Thomas Ursby
}

MAX IV Laboratory, Lund University, P.O.B. 118, SE-221 00 Lund, Sweden

oskar.aurelius@maxiv.lu.se

As the first macromolecular crystallography (MX) beamline at a fourth-generation synchrotron source, BioMAX [1] is also the first MX beamline at the MAX IV Laboratory. The primary usage case has targeted high sample throughput and robustness, using the performance of the MAX IV source to achieve a $20 \times 5 \mu \mathrm{m}^{2}$ beam focus with few beam shaping elements. Operation of the KB-mirror pair used for focusing, is automated to also deliver a defocused beam size of 50 x 50 or $100 \times 100 \mu \mathrm{m}^{2}$. Using a double crystal $\mathrm{Si}(111)$ monochromator, an energy range of 5 to $25 \mathrm{keV}$ is user accessible and a photon flux of $5 \times 10^{12}$ photon/s is routinely achieved at a ring current of $250 \mathrm{~mA}$ and a photon energy of $13 \mathrm{keV}$.

The beamline endstation has been based around well-established components such as the MD3 diffractometer from Arinax, a Dectris Eiger 16M photon counting detector and an IRELEC ISARA sample changer. The beamline is accommodating to a wide range of experiment types, including: cryogenic data collection, humidity-controlled room temperature data collection, optimised SAD/MAD capability, serial crystallography by fixed-target supports or injectors [2], helical data collection, rapid-feedback mesh scans and with a suite of auto-processing pipelines.

Also associated with the beamline is the FragMAX fragment screening program at MAX IV [3]. Beamline control is provided through the web-technology based MXCuBE3 [4] and with the ISPyB database [5] for LIMS functionality via the EXI user interface.

[1] Ursby, T., Åhnberg, K., Appio, R., Aurelius, O., Barczyk, A., Bartalesi, A., Bjelčić, M., Bolmsten, F., Cerenius, Y., Doak, R. B., Eguiraun, M., Eriksson, T., Friel, R. J., Gorgisyan, I., Gross, A., Haghighat, V., Hennies, F., Jagudin, E., Norsk Jensen, B., Jeppsson, T., Kloos, M., Li-donSimon, J., de Lima, G. M. A., Lizatovic, R., Lundin, M., Milan-Otero, A., Milas, M., Nan, J., Nardella, A., Rosborg, A., Shilova, A., Shoeman, R. L., Siewert, F., Sondhauss, P., Talibov, V., Tarawneh, H., Thånell, J., Thunnissen, M., Unge, J., Ward, C., Gonzalez, A. \& Mueller, U. (2020). J. Synchrotron Radiat. 27, 1415. DOI:10.1107/s1600577520008723

[2] Shilova, A., Lebrette, H., Aurelius, O., Nan, J., Welin, M., Kovacic, R., Ghosh, S., Safari, C., Friel, R. J., Milas, M., Matej, Z., Högbom, M., Brändén, G., Kloos, M., Shoeman, R. L., Doak, B., Ursby, T., Håkansson, M., Logan, D. T. \& Mueller, U. (2020). J. Synchrotron Radiat. 27, 1095. DOI: $10.1107 / \mathrm{S} 1600577520008735$

[3] Lima, G.M.A., Talibov, V.O., Jagudin, E., Sele, C., Nyblom, M., Knecht, W., Logan, D.T., Sjögren, T. \& Mueller, U. (2020). Acta Crystallogr. D. 76, 771. DOI: $10.1107 / \mathrm{S} 205979832000889 \mathrm{X}$

[4] Mueller, U., Thunnissen, M., Nan, J., Eguiraun, M., Bolmsten, F., Milàn-Otero, A., Guijarro, M., Oscarsson, M., de Sanctis, Daniele. \& Leonard, G. A. (2017). Synchrotron Radiat. News 30, 22. DOI: 10.1080/08940886.2017.1267564

[5] Delagenière, S., Brenchereau, P., Launer, L., Ashton, A. W., Leal, R., Veyrier, S., Gabadinho, J., Gordon, E. J., Jones, S. D., Levik, K. E., McSweeney, S. M., Monaco, S., Nanao, M., Spruce, D., Svensson, O., Walsh, M. A. \& Leonard, G. A. (2011). Bioinformatics 27 (22), 3186. DOI: $10.1093 /$ bioinformatics/btr535

Keywords: Macromolecular crystallography; Beamline 